\title{
Nombre élevé stable de cas de mauvais traitements d'enfants
}

\section{Markus Wopmann}

Dr med., groupe de travail pour la protection de l'enfant, médecin-chef Klinik für Kinder und Jugendliche Kantonsspital Baden

En 2015, pour la $7^{\mathrm{e}}$ année consécutive, le nombre d'enfants pris en charge dans les cliniques pédiatriques suisses, en ambulatoire ou stationnaire, suite à de mauvais traitements avérés ou suspectés a été enregistré. Cette année, ce sont les données de 20 des 26 cliniques pédiatriques qui ont pu être étudiées, ce qui représente un feed-back de $77 \%$ des cliniques. Encore une fois, toutes les grandes et moyennes cliniques pédiatriques ont transmis leurs cas, si bien que le nombre de cas que nous avons répertorié représente une très grande partie du nombre de mauvais traitements que les cliniques pédiatriques suisses ont pris en charge durant l'année écoulée.

\section{Résultats}

1388 cas au total ont été signalés par les 20 cliniques. Les chiffres d'une clinique qui avait annoncé l'année précédente 23 cas ne sont cette année pas disponibles, si bien que le nombre de cas de 2015 correspond certainement à celui de l'année 2014. Les chiffres de toutes les cliniques participantes sont restés quasiment semblables à ceux de l'année précédente, de sorte qu'il est possible qu'un certain plateau (malheureusement fortement élevé) ait été atteint. La répartition dans les différents sous-groupes donne les chiffres suivants:

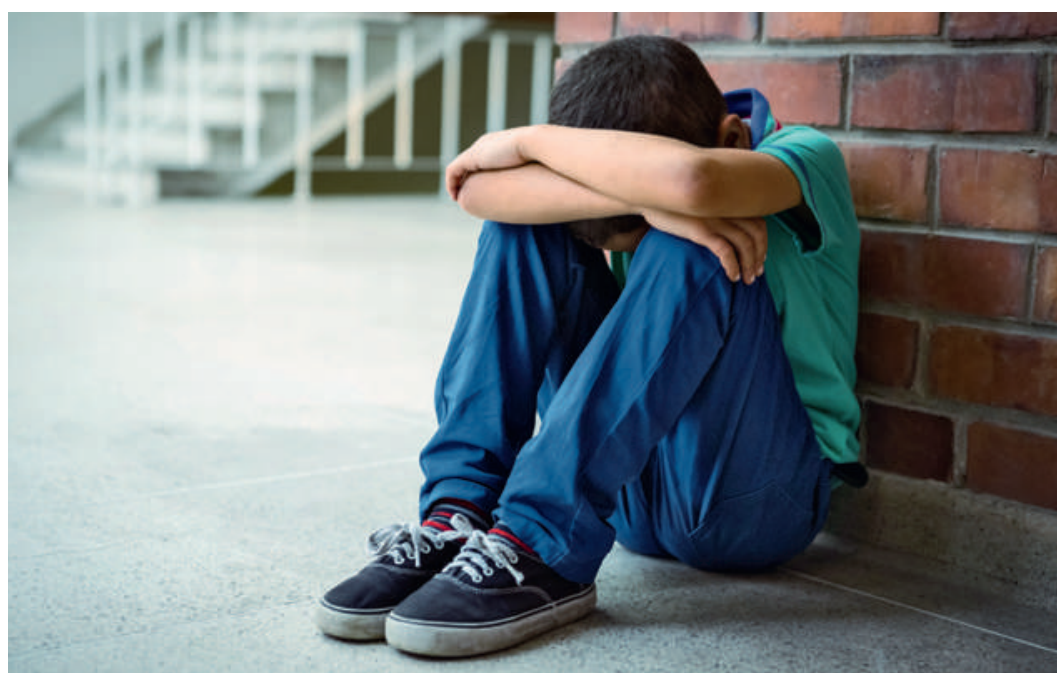

La proportion d'enfants ayant subi de la maltraitance psychique a progressée.

\begin{tabular}{lrr}
\hline Maltraitance physique & 393 & $(28,3 \%)$ \\
\hline Négligence & 277 & $(20,0 \%)$ \\
\hline Maltraitance psychique & 432 & $(31,1 \%)$ \\
\hline Abus sexuel & 276 & $(19,9 \%)$ \\
\hline Syndrome de Münchhausen par procuration & 10 & $(0,7 \%)$ \\
\hline
\end{tabular}

A nouveau, la proportion d'enfants ayant subi de la maltraitance psychique a progressée, si bien que cette forme de maltraitance constitue à présent la plus grande partie des maltraitances totales. Cela est vraisemblablement imputable au fait que de plus en plus de cliniques pédiatriques sont impliquées dans des investigations concernant des cas de violences domestiques, dans lesquelles en effet beaucoup d'enfants sont fortement atteints, au minimum psychologiquement, par la violence entre les parents. Près d'un quart des enfants maltraités étaient âgés de moins de 2 ans, $45 \%$ avaient moins de 6 ans. Avec 43\% de garçons et 57\% de filles, la répartition selon le sexe est presque égale.

\begin{tabular}{lrr}
\hline \multicolumn{3}{l}{ Répartition des sous-groupes selon le sexe } \\
\hline & Garçons & Filles \\
\hline Maltraitance physique & $56,7 \%$ & $43,3 \%$ \\
\hline Négligence & $44,4 \%$ & $55,6 \%$ \\
\hline Maltraitance psychique* & $46,3 \%$ & $53,2 \%$ \\
\hline Abus sexuel & $17,8 \%$ & $82,2 \%$ \\
\hline
\end{tabular}

* Dans $0,5 \%$ des cas, le sexe de l'enfant n'a pas été enregistré.

Encore une fois, la part des garçons battus est plus grande que celle des filles battues, alors qu'en ce qui concerne les autres formes de maltraitance, la proportion est plus élevée pour les filles, avec en premier lieu les abus sexuels, pour lesquels cette année pratiquement cinq fois plus de filles que de garçons ont été concernées.

\begin{tabular}{|c|c|c|}
\hline \multicolumn{3}{|c|}{ Certitude du diagnostic* } \\
\hline Sûr & 842 & $(60,7 \%)$ \\
\hline Probable & 298 & $(21,5 \%)$ \\
\hline Pas clair & 248 & $(17,2 \%)$ \\
\hline
\end{tabular}

Le diagnostic de mauvais traitements psychiques a été, 
avec 75\%, classé comme le plus sûr, la maltraitance physique semble certaine dans $59 \%$ des cas, la négligence dans $61 \%$ des cas. Concernant les abus sexuels, la certitude du diagnostic était donnée seulement dans $42 \%$ des cas.

\begin{tabular}{lrr}
\hline Auteur: relation avec I'enfant & & \\
\hline Famille & 1016 & $(80,3 \%)$ \\
\hline Connu de l'enfant & 166 & $(12,0 \%)$ \\
\hline Extra-familial & 33 & $(2,4 \%)$ \\
\hline Inconnu & 73 & $(5,3 \%)$
\end{tabular}

En outre, les maltraitances psychiques et les négligences ont lieu pratiquement dans tous les cas au sein du noyau familial, les maltraitances physiques dans $3 / 4$ des cas, les abus sexuels dans $46 \%$ des cas. $20 \%$ des agressions sexuelles sont perpétrées par un étranger ou par un auteur inconnu.

\begin{tabular}{llr}
\hline Auteur: sexe & & \\
\hline Masculin & 639 & $(46,0 \%)$ \\
\hline Féminin & 350 & $(25,2 \%)$ \\
\hline $\begin{array}{l}\text { Masculin et féminin (en général } \\
\text { les deux parents) }\end{array}$ & 281 & $(20,2 \%)$ \\
\hline Inconnu & 180 & $(8,6 \%)$ \\
\hline
\end{tabular}

La proportion d'auteurs d'abus sexuel de sexe masculin se situe, avec presque $85 \%$, dans la cadre des autres études nationales ou internationales. Les cas de négligence étaient le fait des mères dans $45 \%$ des cas, et dans $39 \%$ des cas les deux parents étaient co-responsables.

\section{0\% des agressions sexuelles sont perpétrées par un étranger ou par un auteur inconnu.}

Pour les maltraitances psychiques et physiques, la part des auteurs masculins était, avec $45 \%$, plus élevée que celle des auteurs féminins. Pour l'ensemble des 10 cas de syndrome de Münchhausen par procuration, ce sont finalement des femmes qui sont répertoriées comme auteur, ce qui correspond à la littérature internationale.

\begin{tabular}{lrr}
\hline Auteur: âge & & \\
\hline Plus de 18 ans & env. 1157 & (env. 83\%) \\
\hline Moins de 18 ans & env. 130 & (env. 9\%) \\
\hline $\begin{array}{l}\text { Moins et plus de 18 ans } \\
\text { (plusieurs auteurs) }\end{array}$ & 8 & $(0,6 \%)$ \\
\hline Age inconnu / pas d'indication & 93 & $(6,7 \%)$ \\
\hline
\end{tabular}

La part des jeunes auteurs est quasiment restée la même. Tout comme ces dernières années, les auteurs mineurs sont, avec plus de $20 \%$, significativement plus présents dans les cas d'abus sexuels que dans les autres formes de maltraitance.

\begin{tabular}{lrr}
\hline Mesures tutélaires & & \\
\hline Déjà engagées par une autre instance & 338 & $(24,4 \%)$ \\
\hline $\begin{array}{l}\text { Signalement fait par le groupe de } \\
\text { protection de l'enfant }\end{array}$ & 338 & $(24,4 \%)$ \\
\hline $\begin{array}{l}\text { Signalement recommandé par le groupe } \\
\text { de protection de l'enfant }\end{array}$ & 91 & $(6,6 \%)$
\end{tabular}

Mesures pénales

Déjà engagées par une autre instance $\quad 286 \quad(20,6 \%)$

Engagées par le groupe de protection

de l'enfant $\quad 85 \quad(6,1 \%)$

Recommandées par le groupe de

protection de l'enfant

$29 \quad(2,1 \%)$

\section{Conclusion}

- Le nombre d'enfants avec cas de maltraitance traités par les cliniques pédiatriques suisses s'est maintenu à un haut niveau inchangé.

- Avec plus de 30\% des cas, c'est la maltraitance psychique qui a été la maltraitance la plus répertoriée par les cliniques pédiatriques. Cela est à considérer très probablement en relation avec les très nombreux cas de violence domestique, dans lesquels les enfants sont touchés, au minimum de manière indirecte.

- Les groupes de protection de l'enfant ont signalé eux-mêmes un cas sur quatre aux autorités de protection de l'enfant et de l'adulte. Ces autorités avaient été exactement autant de fois impliquées au préalable. Cela s'explique par le fait que les mauvais traitements d'enfants - sauf en ce qui concerne les abus sexuels - se perpètrent souvent dans des familles socialement défavorisées.

- En 2015, un enfant est décédé dans une clinique pédiatrique suisse suite à de mauvais traitements physiques. Cet enfant avait moins d'un an. Pour 250 autres enfants de moins d'un an (ce qui correspond à $18,1 \%$ de tous les enfants concernés!), le diagnostic de maltraitance a été posé. Cela significie que la part des très petits enfants est de nouveau épouvantablement haute.

\section{Crédit photo}

(c) Wavebreakmediamicro | Dreamstime.com

Traduction: Secrétariat SSP 